\title{
DETERMINAÇÃO DO TEOR DE EDTA REMANESCENTE EM SOLUÇÕES DE LIMPEZA DE EQUIPAMENTOS INDUSTRIAIS
}

\author{
Arnaldo Antonio Rodella* \\ Departamento de Ciências Exatas, Escola Superior de Agricultura Luiz de Queiroz, Universidade de São Paulo, CP 9, \\ 13418-900 Piracicaba - SP, Brasil
}

Recebido em 10/5/06; aceito em 16/8/06; publicado na web em 26/3/07

\begin{abstract}
DETERMINATION OF EDTA CONTENT IN CLEANING SOLUTIONS AFTER THEIR USE IN INDUSTRIAL EQUIPMENTS. Cleaning solutions containing EDTA are widely employed to remove incrustation despite of the costs involved. The free content of EDTA in commercial solutions may be determined by mixing an aliquot to ammonium oxalate and using a standard calcium solution as titrant. The end-point is detected by the formation of insoluble calcium oxalate after all EDTA is complexed. A system for turbidimetric detection of the end-point was envisaged to substitute the visual detection, which impaired the analyses of dark samples. The proposed method was tested with real samples and good accuracy and precision was obtained.
\end{abstract}

Keywords: EDTA analysis; turbidimetric end-point; industrial cleaning solutions.

\section{INTRODUÇÃO}

A ocorrência de incrustações em equipamentos industriais representa um sério inconveniente, pois prejudicam ou bloqueiam o fluxo de materiais através de tubulações, dificultam a troca de calor entre superfícies e exigem interrupções periódicas de trabalho para limpeza. As incrustações são acúmulos de material sólido nas superfícies internas de tubulações, tanques e caldeiras, sobretudo como resultado da precipitação de sais inorgânicos, em geral sais de cálcio. Um constituinte comum nas incrustações denominadas duras é o sulfato de cálcio, $\mathrm{CaSO}_{4} \cdot 2 \mathrm{H}_{2} \mathrm{O}$, que predomina nos depósitos em colunas das destilarias de álcool. Na fabricação de açúcar, o problema ocorre com maior intensidade nos tubos das calândrias dos evaporadores de caldo e, neste caso, a composição das incrustações é mais complexa ${ }^{1}$.

A remoção de incrustações em casos mais simples pode ser efetuada mecanicamente com escovas e jatos de água, ou então por meio de equipamentos apropriados, demandando tempo e mão-deobra. Produtos de limpeza também são comumente usados, sendo o ácido nítrico apropriado para remoção de depósitos carbonatados, enquanto agentes complexantes são indicados para incrustações contendo íons silicato e sulfato. O EDTA é empregado em diversos produtos, em geral associado a agentes surfactantes, ou adicionado à água de alimentação de caldeiras como agente preventivo. Estima-se que na Europa sejam usadas anualmente cerca de $215 \mathrm{t}$ de EDTA como agente desincrustante ${ }^{2}$.

O EDTA apresenta custo relativamente elevado sendo inclusive preconizada sua recuperação pela técnica de eletroredução, para separar íon metálicos de seus complexos com EDTA, o qual seria reutilizado como agente de limpeza ${ }^{3}$. Mais comumente, busca-se a simples reutilização de agentes desincrustantes e a eficiência desse processo exige determinar a concentração de EDTA não complexado no material usado.

Um método preconizado para determinação de EDTA em sabões e detergentes tem sido aplicado para a finalidade anteriormente descrita e baseia-se na adição de solução de oxalato de amônio à amostra em meio fortemente alcalino, seguindo-se titulação com solução padrão de cálcio. O íon cálcio adicionado através do titulante

\footnotetext{
*e-mail: aarodell@esalq.usp.br
}

é complexado pelo EDTA eventualmente presente e, uma vez que este é consumido, o primeiro excesso de íon $\mathrm{Ca}^{+2}$ adicionados reage com o íon oxalato e produz turvação pela formação de precipitado de oxalato de cálcio. Isso ocorre porque a afinidade do íon $\mathrm{Ca}^{+2}$ pelo EDTA suplanta sua tendência em ser precipitado pelo íon oxalato:

$$
\begin{array}{ll}
\mathrm{Ca}^{2+}+\mathrm{EDTA}^{4-} \leftrightarrow[\mathrm{CaEDTA}] & \mathrm{K}_{\mathrm{f}}=4,910^{10} \\
\mathrm{Ca}\left(\mathrm{COO}^{-}\right)_{2} \leftrightarrow \mathrm{Ca}^{2+}+2 \mathrm{COO}^{-} & \mathrm{K}_{\mathrm{ps}}=1,310^{-8}
\end{array}
$$

Esse procedimento é adotado pela AATCC - "American Association of Textile Chemists and Colorists"4 e pelo "Texas Building and Procurement Commission"5.

Depois de utilizadas, as soluções desincrustantes normalmente apresentam coloração escura e contêm material em suspensão. Nessas condições tornar-se-ia extremamente difícil perceber visualmente o precipitado de oxalato de cálcio, responsável pela turvação e indicativo do ponto final da titulação. Na verdade, o método analítico em questão tem seu uso restrito a amostras com cor inferior a 500 na escala APHA ("American Public Health Association").

O objetivo do presente trabalho foi propor um sistema para detecção turbidimétrica do ponto final de titulação de EDTA com solução padrão de $\mathrm{Ca}^{+2}$, usando o íon oxalato como indicador, na análise de produtos comerciais após sua utilização na limpeza de incrustações em equipamentos industriais.

\section{MATERIAL E MÉTODOS}

\section{Reagentes}

Solução de oxalato de amônio $30 \mathrm{~g} \mathrm{~L}^{-1}$; solução padrão 0,015 mol L-1 $\mathrm{Ca}^{+2}$ : preparada a partir do $\mathrm{sal} \mathrm{CaCl}_{2}$ e padronizada com solução de EDTA 0,01 mol L $\mathrm{L}^{-1}$, empregando-se Calcon como indicador e solução $0,5 \mathrm{~mol} \mathrm{~L}^{-1} \mathrm{NaOH}$.

\section{Equipamentos}

Para substituir a detecção visual do ponto final de titulação empregou-se um conjunto formado por espectrofotômetro (Shimadzu 
UV-1200), bomba peristáltica (Ismatec IPC 8), agitador magnético e bureta (Figura 1). A solução de amostra, contida em béquer, mantida sob agitação constante, é continuamente circulada por uma cubeta de fluxo contínuo colocada em espectrofotômetro. A velocidade de circulação da amostra não é exatamente crítica, devendo ser suficientemente rápida para obtenção de leituras estáveis em um período de tempo relativamente curto.

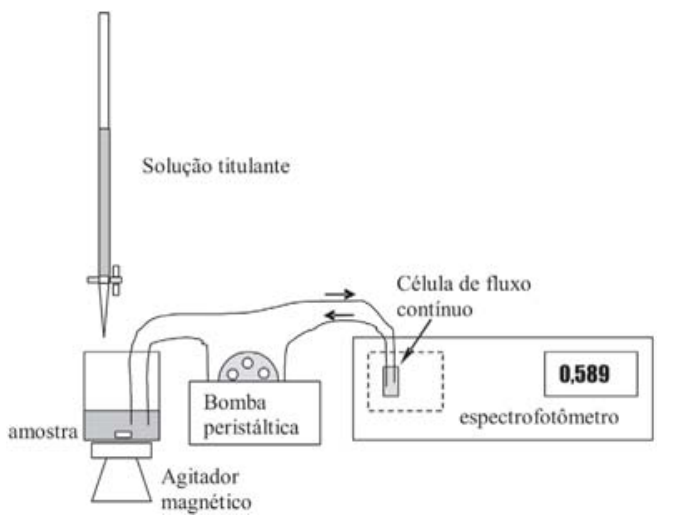

Figura 1. Sistema proposto para a detecção espectrofotométrica do ponto final de titulação para determinação de EDTA na análise de produtos desincrustantes comerciais

\section{Procedimento}

Uma massa de solução de amostra exatamente conhecida, de cerca de $8 \mathrm{~g}$, foi transferida para béquer de $250 \mathrm{~mL}$. Considerou-se a fase líquida da amostra, deixando decantar o material suspenso eventualmente presente ou efetuando a centrifugação da amostra. Adicionou-se cerca de $100 \mathrm{~mL}$ de água destilada, $20 \mathrm{~mL}$ de solução de oxalato de amônio $30 \mathrm{~g} \mathrm{~L}^{-1} \mathrm{e}$, quando necessário, ajustou-se o pH a 11 com solução de $\mathrm{NaOH}$, uma vez que soluções de limpeza já contêm $\mathrm{NaOH}$ em concentrações elevadas.

Circulou-se a solução de amostra e reagentes pelo sistema descrito anteriormente e observou-se o valor de absorbância obtido a $400 \mathrm{~nm}$. Amostras com absorbância muito elevada (acima de 1) foram diluídas no próprio sistema a valores de absorbância inferiores a 0,8 , adicionando-se água destilada e deixando-se homogeneizar. Evidentemente o valor absoluto da absorbância não é importante. Nas amostras onde se exigia diluição muito elevada, pesou-se uma massa menor para análise. Após a adição de cada incremento de volume de titulante foram necessários apenas alguns segundos para que a leitura de absorbância no espectrofotômetro se estabilizasse e pudesse ter seu valor registrado. Os incrementos de volume de titulante foram adicionados em número suficiente para se obter uma curva de titulação. $\mathrm{O}$ ponto final de titulação foi obtido a partir da intersecção dos segmentos de reta ajustados aos trechos iniciais e finais da curva (Figura 2).

\section{RESULTADOS E DISCUSSÃO}

O sistema proposto forneceu leituras de absorbância estáveis e pontos finais de titulação facilmente determinados quando testado em amostras reais de produtos usados na limpeza de equipamentos industriais (Tabela 1). As amostras A-1 a A-4 e F-1 a F-5 foram coletadas durante o transcorrer de uma operação de limpeza e evidenciam o progressivo consumo de EDTA durante o processo. $\mathrm{O}$ índice denominado valor quelante ("chelating value") é comumente empregado na avaliação de soluções contendo EDTA. De maneira geral, o teor de EDTA em muitas das amostras foi relativamente baixo, indicando consumo do agente durante o processo de limpeza.
Tabela 1. Resultados da determinação de EDTA em amostras de produtos desincrustantes comerciais após sua utilização na limpeza de equipamentos

\begin{tabular}{lccc}
\hline Amostra* & $\mathrm{g} \mathrm{Na}_{4}$ EDTA/kg** & $\begin{array}{c}\text { Valor quelante } \\
\mathrm{g} \mathrm{CaCO}_{3} / \mathrm{kg}\end{array}$ & $\begin{array}{c}\text { Recuperação*** } \\
\text { EDTA }(\%)\end{array}$ \\
\hline A-1 & $1,15 \pm 0,018$ & 0,31 & 16,0 \\
A-2 & $0,56 \pm 0,012$ & 0,15 & 7,7 \\
A-3 & $0,00 \pm 0,000$ & 0,00 & 0,0 \\
A-4 & $0,00 \pm 0,000$ & 0,00 & 0,0 \\
B & $0,06 \pm 0,003$ & 0,02 & 1,0 \\
C & $0,04 \pm 0,003$ & 0,01 & 0,5 \\
D-1 & $0,01 \pm 0,000$ & 0,00 & 0,0 \\
D-2 & $1,46 \pm 0,042$ & 0,39 & 20,1 \\
D-3 & $0,57 \pm 0,015$ & 0,15 & 7,7 \\
D-4 & $0,02 \pm 0,001$ & 0,00 & 0,0 \\
E-1 & $0,82 \pm 0,022$ & 0,22 & 11,3 \\
E-2 & $0,95 \pm 0,033$ & 0,25 & 12,9 \\
E-3 & $1,04 \pm 0,029$ & 0,28 & 14,4 \\
E-4 & $0,42 \pm 0,030$ & 0,11 & 5,7 \\
E-5 & $0,22 \pm 0,013$ & 0,06 & 3,1 \\
F-1 & $4,42 \pm 0,118$ & 1,18 & 60,8 \\
F-2 & $0,49 \pm 0,022$ & 0,13 & 6,7 \\
F-3 & $0,25 \pm 0,012$ & 0,07 & 3,6 \\
F-4 & $0,17 \pm 0,008$ & 0,05 & 2,6 \\
F-5 & $0,04 \pm 0,003$ & 0,01 & 0,5 \\
\hline
\end{tabular}

* letras indicam locais e números indicam seqüência de amostragem; ** média e desvio padrão de três determinações; *** calculada a partir do teor de EDTA no produto comercial antes de seu uso

Em diversas amostras a quantidade de EDTA remanescente era nula, observando-se então uma elevação constante da absorbância desde o primeiro incremento de titulante adicionado, sem que um ponto de inflexão pudesse ser definido (Figura 2b).
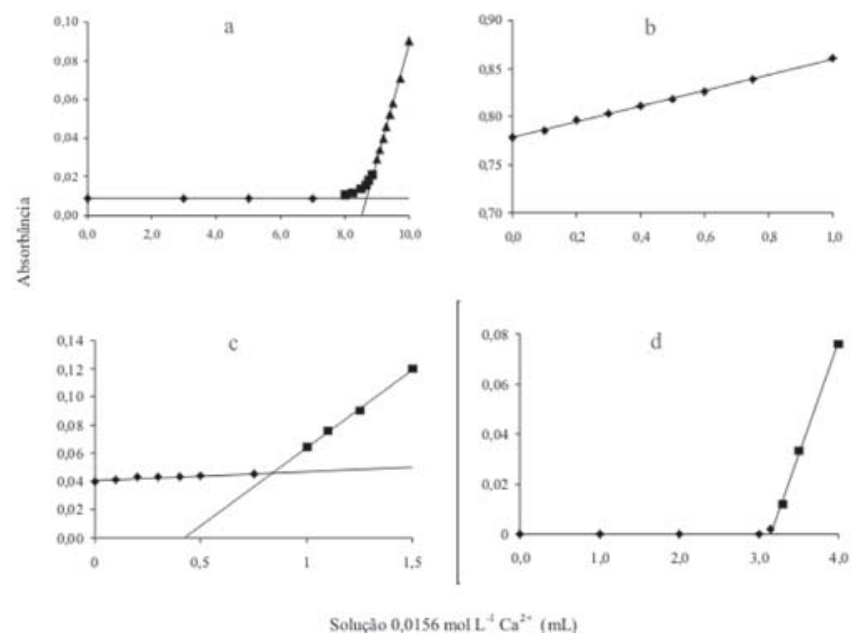

Figura 2. Curvas de titulação típicas: a e c correspondem respectivamente a teores relativamente elevado e baixo de EDTA remanescente em amostras; $b$ amostra que não contém EDTA. A curva d corresponde à titulação de $5 \mathrm{~mL}$ de EDTA 0,01 $\mathrm{mol} \mathrm{L}^{-1}$

Desvios padrões relativos entre 1,56 e $7,50 \%$ atestam a precisão de determinações pelo procedimento proposto. A titulação de uma solução padrão de EDTA confirmou a exatidão da técnica proposta (Figura 2d). Na titulação de $5 \mathrm{~mL}$ de solução $0,01 \mathrm{~mol} \mathrm{~L}^{-1}$ de EDTA gastou-se $3,16 \mathrm{~mL}$ de solução $0,0156 \mathrm{~mol} \mathrm{~L}^{-1} \mathrm{Ca}^{+2}$. 
Um teste de recuperação foi conduzido com a amostra A-4 que, conforme evidenciado na Tabela 1, não apresentava teor de EDTA mensurável. Quantidades crescentes de EDTA foram adicionadas a diversas alíquotas de 2,00 $\mathrm{g}$ da amostra A-4 e procedeu-se à determinação de EDTA conforme descrito anteriormente.

Os resultados indicaram que a primeira porção de EDTA adicionada não foi detectada (Figura 3). Isso ocorreu porque a amostra A-4, além de não conter concentração alguma de EDTA remanescente, apresentava componentes passíveis de serem complexados pelo EDTA adicionado.

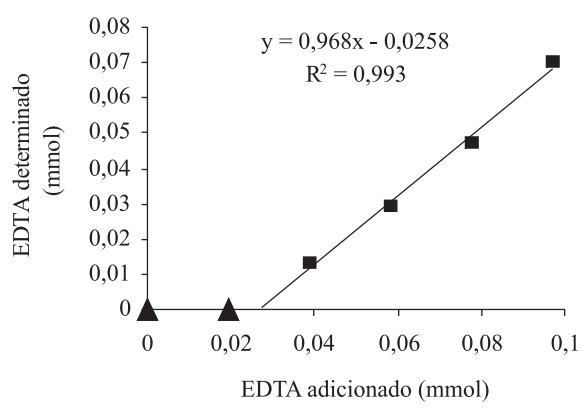

Figura 3. Recuperação do EDTA adicionado à amostra de solução desincrustante que não continha EDTA livre (amostra A-4)

A equação de regressão entre valores determinados (y) e valores adicionados (x) evidencia uma recuperação de 96,8\% do EDTA adicionado (Figura 3). Outra informação relevante do ponto de vista prático é que uma massa de $2,00 \mathrm{~g}$ de amostra foi capaz de complexar 0,026 mmol de EDTA. Após serem usadas, as soluções desincrustantes normalmente ainda apresentam concentrações elevadas de $\mathrm{NaOH}$ e muitas vezes pretende-se adicionar EDTA às mesmas. Essa prática deve levar em conta a possibilidade do material conter concentrações significativas de impurezas capazes de consumir EDTA. No caso da amostra A-4 esse consumo seria de $5,05 \mathrm{~g}$ de $\mathrm{Na}_{4}$ EDTA por $\mathrm{kg}$ de material.

A detecção turbidimétrica do ponto final de titulação de EDTA com solução padrão de cálcio mostrou ser eficaz para contornar as incertezas associadas à dificuldade de observação visual do precipitado de oxalato de cálcio em amostra escuras e turvas.

\section{AGRADECIMENTOS}

À técnica de laboratório J. Truffi pela execução das análises.

\section{REFERÊNCIAS}

1. Gloria, N. A.; Rodella, A. A.; Brasil Açucareiro 1973, 82,12.

2. Institute For Health and Consumer Protection - European Chemicals Bureau; Edetic Acid (EDTA) Summary Risk Assessment Report Final Report, Alemanha, 2004.

3. Huang, C. P.; Hsu M.; Miller, P.; J. Environ. Eng. 2000, 126, 919.

4. http://www.aatcc.org/Technical/Test_Methods/scopes/tm149.cfm, acessada em Março 2006.

5. http://www.tbpc.state.tx.us/spec_lib/180-34-01C.pdf, acessada em Março 2006. 